\title{
DESARROLLO DE VARIOS SISTEMAS EXPERTOS DE APOYO A LA ENSEÑANZA DE DISCIPLINAS TÉCNICAS EN LENGUA INGLESA
}

\author{
M. Isabel González Pueyo. C.P.S. Universidad de Zaragoza
}

\begin{abstract}
En este artículo se presenta un sistema multimedia para el aprendizaje del léxico inglés para fines específicos dentro de un paradigma de enseñanza de lenguas centrado en el estudiante -Student-centered communicative and collaborative learning- y que se basa en los principios siguientes:

- El aspecto comunicativo del lenguaje.

- El uso auténtico de la lengua.

- La enseñanza centrada en el estudiante.

- La autonomía del aprendizaje.

- El proceso activo del aprendizaje y la colaboración del estudiante en el mismo, partiendo de sus propios conocimientos y habilidades.

Se comentan los tres principios que deben regir cualquier planificación de todo material software para un curso de lenguas centrado en el alumno, a saber: actividades, presupuestos lingüísticos y diseño del material.
\end{abstract}

\section{INTRODUCCIÓN}

Uno de los problemas que el profesor de inglés técnico en escuelas y facultades de ingeniería encuentra, es el gran número de palabras técnicas que los alumnos deben conocer, con el agravante del reducido tiempo de instrucción disponible y el hecho de que puedan usar la lengua inglesa en una gran variedad de situaciones, no contempladas en el programa docente. Se hace, por tanto, imprescindible buscar soluciones alternativas que permitan al alumno desarrollar estrategias cognitivas de exploración y de descubrimiento, que les ayuden a ser capaces de planificar, controlar y evaluar su propio proceso de aprendizaje. Se necesita un material que cubra estas necesidades y que permita al alumno trabajar a su propio ritmo, según su nivel y preparación, y en el campo que más le interese.

Una solución a este problema podría ser la elaboración de un material multimedia capaz de cubrir las necesidades específicas de los alumnos de carreras técnicas; que permita al profesor desarrollar su práctica docente desde la perspectiva del aprendizaje autónomo 
necesario para que los estudiantes puedan realizar un aprendizaje continuo de la lengua inglesa. Con tal propósito, y con el fin de aprovechar al máximo las infinitas posibilidades que las nuevas tecnologías ofrecen, y la creciente capacidad del alumno para su utilización, nos propusimos, con ayuda de un proyecto, elaborar el material multimedia necesario para el aprendizaje del léxico inglés especializado, adecuado a las necesidades de los alumnos de carreras técnicas. En este artículo se describen estos programas y los motivos que nos llevaron a su elaboración.

Al diseñar el material se han tenido en cuenta las características específicas de las asignaturas, y las actividades se han desarrollado según las teorías del aprendizaje de lenguas centrado en el alumno, cuyo exponente teórico es el "constructivismo". En estas propuestas el ordenador es considerado como instrumento que media en los procesos de enseñanza y aprendizaje, según el marco de la concepción triádica de la interacción educativa (Alumnado, Profesorado y Software) propuestos por Richards y Rodgers (1986), y aplicado a la evaluación de los programas de CALL por Hubbard (1992) y otros (Ruschoff, 1993; Armstong y Yetter-Vassot, 1994), quienes hacen especial hincapié en el hecho de que las capacidades de las nuevas tecnologías no deben condicionar los principios de la práctica educativa, sino que son las consideraciones de tipo pedagógico y lingüístico las que deben guiar la adopción de las nuevas tecnologías en la clase de lenguas.

Es indudable que estamos ante un nuevo paradigma de la enseñanza, y que, por tanto, no existen todavía modelos suficientemente contrastados y probados que puedan orientar al didacta y al investigador; tampoco hay una agenda común para la investigación y el desarrollo de la enseñanza de lenguas asistida por ordenador (CALL), de manera que, los autores de CALL suelen extraer sus conclusiones teóricas a partir de orientaciones prácticas. Como señalaba Wenger (1987: 7):

we are approaching a point where CALL and multimedia can decide to remain essentially engineering applications of technology for specific learning tasks and problems. There are several theoretical tracks which are potentially open. One lies in the direction of education. Another lies towards expert systems and cognitive science, where work on scaffolding, apprenticeship and constructivist models of learning are currently arousing significantly.

A esta imprecisión de los modelos se añade cierta confusión en la terminología. CALL se define, en general, como Computer-Aided Language Learning (o Enseñanza y Aprendizaje Asistidos por Ordenador EAAO), y engloba otros términos como ON-LINE teaching C\&IT (Computers and Information Technology), IBL (Internet Based Learning), RBL (Resource-Based Learning): todos ellos referidos realmente a lo que es la enseñanza/ aprendizaje en sistemas multimedia.

Otro problema, no menos importante, es el gran desajuste que existe entre los materiales multimedia existentes y las necesidades reales y concretas en situaciones determinadas. Constantemente se pide desde CALL la elaboración de programas que sean más comunicativos: "The content of courseware (...) must come into line with current views about real communication and language use by language learners" (Sanders y Kenner,1984: 38). Sin embargo, como Egbert y Hanson-Smith (1999: 4) señalan, "there is not much ESL software that suits their purposes". El problema está en que, como Meskill comenta (1999: 11-12), "while education has at its disposal technologies that were developed using the funds of other sectors, the down side is that the technology was not specifically designed for what we know serves the best interests of learners".

La elaboración de programas para la enseñanza-aprendizaje que aquí se presentan, surge de esta necesidad y trata, en parte, de solucionar estos problemas. En la elaboración

Odisea, $n^{0} 2,2002$ 
del material se han tenido en cuenta los siguientes criterios didácticos: (i) enfoque comunicativo basado en tareas relacionadas con las disciplinas de los estudiantes; (ii) material personalizado, diseñado especialmente para el alumno; (iii) enseñanza-aprendizaje basada en el contenido, que está relacionado con la disciplina del estudiante. Partimos de que la adquisición de conocimientos, habilidades y estrategias está relacionada con escenarios o contextos de uso. Por tanto, se ha intentado presentar los términos en unos contextos capaces de asegurar un buen proceso de aprendizaje.

\section{METODOLOGÍA}

En primer lugar se ha hecho un análisis de las necesidades específicas de los estudiantes de ingeniería. Para ello se elaboró y distribuyó una encuesta entre 80 estudiantes de ingeniería y 45 egresados que están trabajando en empresas en diversas ciudades españolas. El propósito de las mismas era averiguar los parámetros siguientes:

- Cantidad de vocabulario estudiado en clase en comparación con el vocabulario que consideran importante en cada especialidad. (Ver gráfica 1)

- Tipo y cantidad de vocabulario utilizado por postgraduados en sus lugares de trabajo. (Ver gráfica 2)

- Grado de aceptación del método. (Ver gráfica 3)

Los resultados estadísticos a estas cuestiones pueden verse en las tablas 1, 2 y 3.

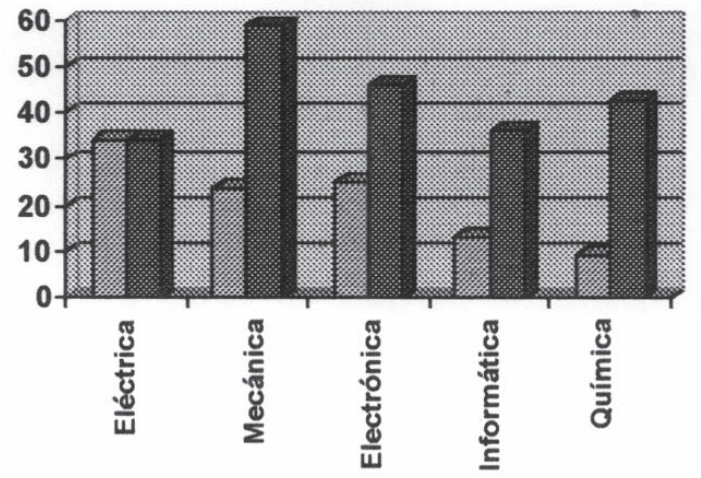

Gráfica 1.

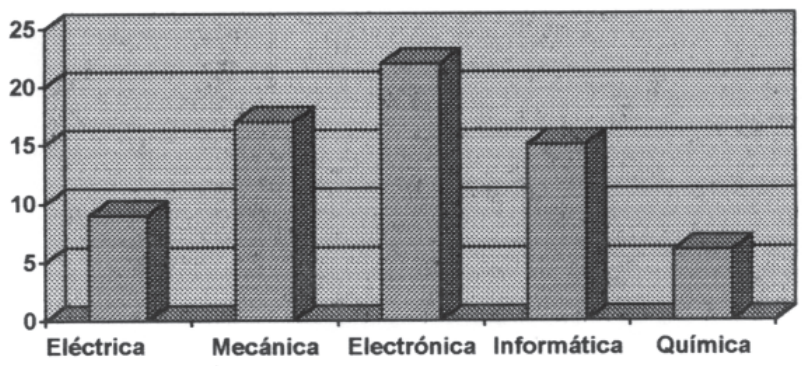

Gráfica 2. 


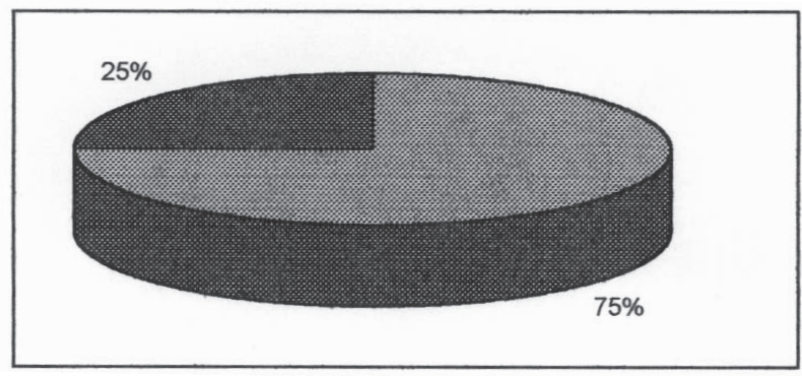

Gráfica 3a. Grado de aceptación del método por estudiantes.

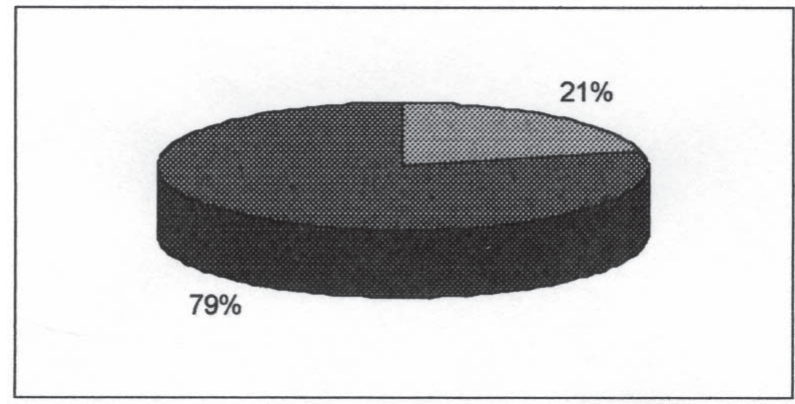

Gráfica 3b. Grado de aceptación del método entre posgraduados.

El análisis mostró un déficit considerable en el aprendizaje del vocabulario, así como un cierto desfase entre el vocabulario que se aprende en clase y el vocabulario que realmente se usa en las distintas especialidades. Asimismo, los datos obtenidos confirmaron la necesidad de introducir un material capaz de satisfacer las necesidades de los alumnos y, que, además, fuera capaz de motivarles e involucrarles en su propio aprendizaje con tareas y ejercicios propios de su campo específico. Este material debería presentar las características siguientes:

- Vocabulario específico en su contexto.

- Situaciones técnicas determinadas y variadas.

- Tareas y ejercicios variados y acordes con la situación presentada.

- Audiciones.

- Algo de refuerzo gramatical.

- Tests de auto-evaluación y control.

Finalmente, el método seguido para desarrollar el material debería basarse en un paradigma de lenguas centrado en el alumno que se articula en los principios siguientes

- El aspecto comunicativo del lenguaje.

- El uso auténtico de la lengua.

- La enseñanza centrada en el estudiante.

- La autonomía del aprendizaje. 
- El proceso activo del aprendizaje y la colaboración del estudiante en el mismo, partiendo de sus propios conocimientos y habilidades.

El material software se ha hecho en colaboración con estudiantes de ingeniería en prácticas. Se pretende con tal colaboración, implicar a los estudiantes en su propio aprendizaje, a la par que aprovechar sus conocimientos y los medios tecnológicos disponibles como un medio creativo para aprender. Se fomenta, así, el aprendizaje autónomo, ya que uno de los objetivos al crear este material es que el alumno pueda utilizarlo como refuerzo. Aunque aplicar la autonomía en el aprendizaje de lenguas "is highly problematic" (Benson y Voller, 1997: 28), estamos de acuerdo con Hurd et al. (2001: 341) en que "autonomy can nevertheless be promoted through careful attention to materials design”. Precisamente, Sussex (1991: 26) nos señala la importancia de diseñar el material en lo que se refiere a la enseñanza de lenguas por ordenador:

At present time rather little work has been done on the question of how teachers become CAL authors: how they objectify their knowledge domains, learning and teaching: how they conceptualise learning materials and learning modes for transfer to the CAL medium: how they achieve this transfer: how the existence and use of CAL media influence theories of CAL and viceversa.

La producción del material se ha llevado a cabo en las etapas siguientes:

- Análisis de datos.

- Recogida de material auténtico.

- Clasificación del vocabulario técnico por campos específicos.

- Preparación de los contenidos, tareas y ejercicios.

- Preparación de los distintos programas o módulos.

- Desarrollo del material software.

\section{DESCRIPCIÓN DEL MÉTODO ELABORADO}

Los programas han sido elaborados sobre la base del constructivismo y las teorías generales sobre cómo se estructura el léxico en la mente, y cómo se adquiere el vocabulario.

\section{El aprendizaje autónomo y el constructivismo}

Es indudable que los programas de CALL se han ido diversificando y modificando, tanto su concepto como la realidad de sus productos, sobre todo tras la implantación de la red, y estamos ya ahora en el punto en que el propio concepto de CALL está siendo reformulado. Esta reformulación está ligada a las teorías del aprendizaje en las que el estudiante autónomo desempeña un papel activo. Así, en vez de reaccionar a los estímulos del profesor, el estudiante tiene la oportunidad de generar sus propias ideas, emplear su imaginación y aprovechar las oportunidades para su aprendizaje (Boud, 1988; Kohonen, 1992; Knowles, 1975; Bongaerts, 1996). Para Rathbone (1971: 100-104, citado en Candy, 1991: 271), el estudiante autónomo es "a self-activated maker of meaning, an active agent in his/her own learning process. He is not one to whom things merely happen; he is the one who, by his own volition, causes things to happen. Learning is seen as the result of his own self-interaction with the world".

En lingüística aplicada esta línea de razonamiento está estrechamente relacionada con la de Halliday (1979) y se encuadra dentro de la teoría lingüística del constructivismo, que 
sostiene que el conocimiento, antes que aprenderse o descubrirse, se construye, mediante la aportación de las experiencias personales del individuo. Como Candy (1991: 254) observa, uno de los pilares centrales del constructivismo es que "individuals try to give meaning to, or construe, the perplexing maelstrom of events and ideas in which they find themselves caught up". En oposición a otras teorías, como el positivismo, el constructivismo cree que los individuos reorganizan sus experiencias para construir el conocimiento. Así, el constructivismo "leads directly to the proposition that knowledge cannot be taught but only learned; that is, constructed, because knowledge is something 'built up by the learner"” (von Glasesfeld y Smock, 1974: xvi, citado en Candy 1991: 270). De la misma manera, el aprendizaje de lenguas no requiere interiorizar una serie de reglas, estructuras y formas, sino que cada individuo aporte su propia experiencia y conocimiento del mundo para poder realizar la tarea correspondiente. El constructivismo, pues, soporta, extiende y cubre aspectos psicológicos de la autonomía que pertenecen al comportamiento del individuo, tales como la motivación, y la auto-estima ( Benson y Voller, 1997: 23).

Sin embargo, la autonomía en el aprendizaje no supone falta de ayuda del profesor. Es más, los profesores desempeñan "a crucial role to play in launching learners into selfaccess in lending them a regular helping hand to stay afloat" (Sheerin, 1997, citado en Benson y Voller, 1997: 63). Sin lugar a dudas, dar el salto, desde un control del aprendizaje, por parte del profesor, a la cuasi-autonomía del alumno, va a entrañar dificultades. Como Candy ha señalado "learner-control is not a single, unitary concept, but rather a continuum along which various instructional situations may be placed" (Candy, 1991: 205).

Estamos de acuerdo con estos autores en que un modo de ayudar al estudiante a alcanzar un grado considerable de autonomía en su propio aprendizaje, es utilizar el ordenador como vehículo de auto-expresión.

\section{El aprendizaje del vocabulario}

Normalmente se reconocen tres estadios que operan cuando se aprende una palabra (Craik y Lockhart, 1972; Anderson, 1990):

1. Reconocimiento de las diversas propiedades de la palabra nueva, tal como su fonología, morfología, sintaxis, semántica, colocación etc.

2. Almacenamiento del léxico interno en redes de relaciones que corresponden a las propiedades arriba descritas.

3. Consolidación del almacenamiento mediante más exposición de la palabra en variedad de contextos que ilustren sus diversas propiedades. De esta manera la palabra se incrusta más fuertemente en la memoria que se requiere para su retención a largo plazo, condición necesaria para un uso receptivo y productivo eficaz.

El proceso natural de adquisición de una palabra (tal como ocurre el la adquisición de L1) consiste en la adquisición gradual de las diversas propiedades de la misma mediante exposiciones en una gran variedad de situaciones auténticas que ilustren sus propiedades varias. Siguiendo estos criterios, en la elaboración de los programas que este artículo describe, se ha intentado agilizar el proceso de aprendizaje del vocabulario técnico, mediante la exposición de los términos en contextos significativos y relevantes para el usuario.

La importancia del contexto en el procesamiento de la información lingüística y perceptiva es indudable. Un número considerable de estudios sobre adquisición de lenguas 
(véase Horst, Cobb y Meara, 1998; Knight, 1994; Krashen, 1989; Nation, 1994) examinan el papel que éste ejerce en la adquisición del vocabulario durante la lectura y las tareas relacionadas con ella. A este respecto Nation (1994) comenta que solamente en los contextos se muestran al completo las características semánticas, sintácticas y colocaciones de la palabra que el sujeto tiene que procesar, ya que ello permite establecer conexiones y asociaciones múltiples con otras palabras, cosa imprescindible para poder recordarla y utilizarla adecuadamente, asegurando así un buen proceso de adquisición. Aunque algunos autores señalan que el vocabulario puede adquirirse también de manera accidental, mediante la presunción de la palabra, ya que el esfuerzo mental que se tiene que realizar al deducir el significado de las palabras contribuye a que éstas se fijen mejor en la memoria (Krashen, 1989), hay otros estudios que han demostrado los beneficios de utilizar glosarios, anotaciones y diccionarios -además del contexto- en la adquisición del vocabulario (por ejemplo, Hulstijn, Hollander y Greidanius, 1996; Knight, 1994; Krantz, 1991; Lomicka, 1998; Lyman-Hager et al, 1993; Chun y Plass, 1996).

Por otra parte, los informes más recientes sobre experimentos de adquisición de vocabulario por ordenador. (Davies, 1989; Chun and Plass, 1996; Lyman-Hager y Davies, 1997; Lomicka, 1998; Mayer, 1997), nos indican que la capacidad del ordenador para controlar y orquestar los diversos medios multi-media -tales como dibujos, sonido, animación, glosarios, diccionarios, vídeo etc.- no han sido conseguidos por ningún otro sistema.

\section{DESCRIPCIÓN DE LOS PROGRAMAS}

Para asegurar la implicación del usuario en el proceso, los programas son interactivos, ya que en ocasiones éste tiene que elegir o contestar al ordenador. Los programas, aunque siguen una estructura similar, son independientes unos de otros: el tipo de actividades que contienen también difieren, ya que se ha procurado aunar la creatividad con la variedad y el contenido. En general el nivel de los programas es medio, aunque alguno de ellos tiene un nivel superior, dependiendo siempre de la dificultad del contenido y de la tarea que se desee hacer. En general los programas contienen:

- Palabras técnicas. Estas siempre aparecen en su contexto específico, con el fin de que el usuario aporte su conocimiento previo y pueda conectar la información nueva con la ya conocida (Prince, 1996).

- Glosarios, que muestran la definición del término en su contexto. Además, el usuario tiene acceso no sólo al significado del término sino también a las estructuras gramaticales propias de la definición, tales como las oraciones de relativo. Se introducen pocos términos de vez, ya que es más efectivo estudiar una palabra con regularidad en sesiones cortas que no en largas (Pimsleur, 1967).

- Traducción de los términos (a veces en varios idiomas) ya que Hulstijn, Hollander y Greidanus (1996) han demostrado que la combinación de palabras con glosarios o diccionario bilingües incrementan el aprendizaje.

- Gráficos, dibujos, animación etc. que muestran el funcionamiento o descripción de los objetos o sistemas. Puesto que las imágenes visuales atraen la atención del usuario con prontitud, asociar el término con su imagen ayuda a una mayor retención de la palabra. Ello contribuye, asimismo, a un proceso mental más profundo (Craik y Lockhart, 1972).

- Textos explicativos, que siempre que ha sido posible, van acompañados de audiciones. 
- Tareas y actividades de carácter interactivo y de dificultad diversa, tales como ejercicios variados, juegos, y finalmente la resolución virtual de un problema concreto o estudio de caso, en el que el usuario tiene que utilizar el léxico aprendido para asegurar su retención a largo plazo (v.g., planificar el sistema de regulación de una casa, o una red local de telecomunicaciones, o jugar en bolsa etc). Esto hace el material "más real" y contribuye al desarrollo de las cualidades cognitivas requeridas en ingeniería. En todo momento se ha procurado seguir el criterio de ir desde tareas receptivas a tareas productivas.

- Sistemas de test, auto-evaluación y control. Todos los módulos contienen algún test de tipo diverso para que el usuario pueda evaluar sus conocimientos.

Los programas cubren los siguientes campos semánticos:

- Ascensores.

Este programa describe las distintas partes y características de los ascensores y su montaje.

- Control.

Este programa muestra mediante tres ejemplos el funcionamiento de sistemas automáticos, actuadores y sensores. El usuario debe realizar un estudio de caso para el calentamiento de una casa.

- Dibujo, imágenes, geometría.

Módulo para que el usuario pueda crear sus propios dibujos a la vez que aprende los términos ingleses de las principales formas geométricas. Está organizado como un sencillo programa de $\mathrm{CAD}$, donde se obligue al usuario a teclear el nombre de la figura geométrica que desea utilizar. De esta forma se cumple la doble función de mostrar un programa de CAD $\mathrm{y}$ aprender términos ingleses de geometría.

- Economía.

Consta de varios programas que permiten adquirir el vocabulario técnico utilizado en contabilidad, impuestos, bolsa, etc. Contiene: juegos pedagógicos, resolución de crucigramas y ayudas.

- Electrodomésticos.

En este programa se describe mediante imágenes y glosarios la estructura y elementos que componen varios electrodomésticos, como el microondas, la cocina vitrocerámica, el horno, la lavadora etc. Se acompaña de tests y un diccionario en varios idiomas, que presenta la particularidad de estar abierto para introducir nuevas palabras.

- Estadística.

Sistema de encuesta de nombre, sexo, edad, profesión, procedencia, etc. El programa informa al usuario del número de veces que ha sdo consultada esa página, y da información sobre distribuciones estadísticas de los datos de las personas apuntadas.

\section{- Física.}

Este programa consiste en la realización de una base de datos de conceptos de la física. Consta de dibujos, definiciones e imágenes, indexados en un sistema de gestión de archivos ágil y eficaz, capaz de sustentar un acceso rápido a los elementos semánticos, los cuales pueden ser modificados, borrados o añadidos por parte del usuario.

- Información.

Base de datos de términos ingleses sobre los siguientes campos científicos: biología, química, informática, tecnología. Añadido a la base de datos hay un sistema de evaluación de los conocimientos del usuario sobre esas materias. El programa desarrollado simula la búsqueda de varios libros en una biblioteca; tiene la estructura de un juego, donde el usuario puede buscar por las principales materias disponibles en una biblioteca varios libros que le ha pedido el sistema.

Odisea, $n^{\circ} 2,2002$ 
- Instrucciones.

Este programa consiste en la realización de varias tareas, como la construcción de una grúa o un semáforo, siguiendo unas instrucciones en inglés paso a paso. Se acompaña de textos.

- Lógica.

Este programa recoge los conceptos básicos de filosofía y ciencia, pensamientos filosóficos y reglas de la lógica, organizados como posters.

- Ordenadores.

Este programa describe el funcionamiento de un ordenador. Texto asociado a imágenes para mostrar las diferentes partes y su funcionamiento. Se acompaña de un glosario y un test de autoevaluación.

- Procesos de fabricación.

Realización de un módulo descriptivo de los principales métodos utilizados en los entornos de fabricación de piezas.

- Vehículos.

Sistema para que el usuario diseñe su propio automóvil. El usuario puede elegir entre un conjunto de chasis, motores, cajas de cambios, sistemas de refrigeración, neumáticos, frenos etc. Una vez ensamblado el automóvil, el sistema verifica sus prestaciones, velocidad máxima, pendiente máxima superable, ángulo de ataque, ángulo de salida etc.

Además hay unos programas de ayuda gramatical complementaria y de autoevaluación y control:

- Gramática para principiantes.

Este módulo constituye una herramienta para aquellos alumnos o usuarios que no han estudiado inglés en ningún momento de su vida académica o cuyos conocimientos son escasos. Contiene 12 unidades que presentan estructuras gramaticales en contexto, con explicaciones en español, ejercicios y tests; el usuario puede ir de una unidad a otra fácilmente y puede revisar sus propios ejercicios. Trabajando adecuadamente el alumno puede alcanzar un cierto nivel para poder seguir el curso de inglés técnico que normalmente se imparte en las escuelas técnicas de ingeniería. Oferta, además, la posibilidad de ser utilizado como material de consulta mientras se corren otros programas.

- Sistema de auto-evaluación y control.

Este sistema sirve tanto para uso individual como para una clase completa. Seleccionando el nivel de inglés que se posee, o el campo semántico que se quiere examinar, el módulo realiza tests para evaluar al alumno, con el número de preguntas deseado y un tiempo de realización. Existen multitud de posibilidades de formación de las pruebas según los criterios seleccionados por el usuario o el profesor para su realización. Una vez realizado el test, el programa permite varias opciones para ver la corrección del mismo: ver todas las preguntas, sólo las falladas, sólo las acertadas, etc. También tiene posibilidad de realizar gráficas que muestren los resultados del alumno en la última prueba realizada, su evolución en las diferentes pruebas que ha realizado, o su posición en cuanto a resultados frente al resto de la clase en que se encuentra ( $\mathrm{si}$ es el caso), por citar sólo algunas de las posibilidades existentes.

\section{CARACTERÍSTICAS DE LOS PROGRAMAS}

La mayoría de esto programas se presentan en soporte CD-ROM, han sido programados en el lenguaje de programación Visual Basic para Windows, y pueden ser 
utilizados tanto como complemento de clase -clase prácticas etc.-, como para el aprendizaje autónomo.

Después de realizar un análisis de los distintos métodos existentes en el mercado, resaltamos las principales características del método que aquí se presenta:

- Cubre un campo -inglés técnico- no cubierto por material software.

- Presenta una estructura didáctica homogénea y de alto rendimiento de los contenidos del programa y de sus diferentes módulos.

- Ofrece un gran número de ejercicios, tomando en la mayoría de los casos situaciones relacionadas con la ingeniería, la ciencia y la técnica.

. Da opción a que el usuario elabore su propio itinerario de aprendizaje, debido a la flexibilidad del sistema modular de los programas.

- Permite al usuario modificar algunos de los parámetros de los programas, como los glosarios, de forma que pueda ir adecuándolos a las necesidades que le surjan.

- Permite la corrección y repetición de los ejercicios tantas veces como fuere necesario. Adicionalmente, el usuario puede ver solamente las respuestas incorrectas, o su totalidad, en función de sus requerimientos.

- Los ejercicios pueden hacerse con o sin control de tiempo, siendo este tiempo almacenado en el cómputo de tareas de cada usuario.

- Muchos de los programas disponen de archivos sonoros para perfeccionamiento de la pronunciación y entonación.

- Los programas son de manejo sencillo y agradable. La temática de los textos se acerca al entorno del usuario, para que sean a la vez útiles y motivadores.

- Presentan un alto grado de autonomía, es decir, que pueden seguirse con la mínima participación de un tutor externo. Aunque la presencia del tutor es siempre una buena ayuda, el objetivo de este proyecto es precisamente asegurar que el curso no dependa excesivamente de un horario de tutorías o de clases presenciales. Para asegurar este punto, se ha dotado al curso de ayudas complementarias -soluciones, textos explicativos, anotaciones, glosarios etc.- y de unos medios de auto-corrección adecuados.

\section{CONCLUSIÓN}

En este artículo se ha presentado la elaboración de material multimedia para el aprendizaje de léxico técnico en lengua inglesa en contextos específicos. Los objetivos que llevaron a su elaboración fueron de orden didáctico y metodológico. Por un lado se hacía imprescindible la creación de un material que se adaptara a las necesidades de los alumnos de carreras técnicas; por otro, ese material debería reunir una serie de requisitos, tales como la motivación y la creatividad. Dado que el objetivo básico es "aprender a aprender", los profesores o tutores de inglés específico no pueden centrarse sólo en enseñar la lengua que se usa en todas las situaciones que los alumnos van a necesitar; por tanto es necesario potenciar que los estudiantes desarrollen estrategias cognitivas que les permitan planificar, evaluar y controlar su propio aprendizaje, así como ofrecerles la posibilidad de poder trabajar a su propio ritmo y en los temas y situaciones que más les interesen. Estas estrategias se pueden utilizar de varias maneras. Así, al diseñar el material, se ha intentado en todo momento que tanto las actividades y tareas, como el contenido, potencie las estrategias requeridas para un aprendizaje autónomo, al tiempo que se ha tratado de concienciar al alumno de qué aprender y cómo se aprende, condición básica para un aprendizaje eficaz. Como ya señalaba Polya (1960: 9) la capacidad general para resolver

Odisea, $n^{\circ}$ 2, 2002 
problemas puede enseñarse. Con tal fin, se han utilizado contextos de uso auténticos, cercanos a la realidad técnica del alumno. Se ha intentado también ir creando una base de datos abierta de términos técnicos, para que el usuario tenga acceso rápido al término, $\mathrm{y}$ pueda crear su propio "diccionario".

Se está ahora elaborando un instrumento de seguimiento del aprendizaje del alumno con este sistema. Sin embargo, los datos que actualmente tenemos, si bien escasos, obtenidos vía encuestas personales y tests de evaluación con varios grupos, nos permiten ser optimistas, ya que se observa un alto grado de aceptación por parte del alumno, así como una mayor retención del vocabulario. Respuestas como "muy satisfactorio", "me he divertido", "he aprendido", han las sido mayoritarias. Por otro lado, al contribuir el alumno de una manera activa en su propio aprendizaje y en la selección de un material cuya utilización va a necesitar, presta más interés en su aprendizaje, ya que está aprendiendo lengua inglesa a la vez que contenido. El hecho de implicar al alumno en un proyecto, también favorece su faceta investigadora y le prepara para poder participar en los proyectos de $\mathrm{I}+\mathrm{D}$, que es uno de los requisitos hoy en día exigidos en la industria.

Esperemos que en un futuro próximo, podamos obtener resultados más amplios y acreditados para poder valorar los beneficios del método.

\section{BIBLIOGRAFÍA}

Anderson, A, y T. Linch. 1988. Listening. Oxford: Oxford University Press.

Armstrong, K. y C. Yetter-Vassot. 1994. "Transforming teaching through technology". Foreign Language Annals 27, 4: 475-486.

Benson, P. y P. Voller. 1997. Autonomy and Independence in Language Learning. London: Longman.

Bongaerts, T. et al. 1996. The Power of CALL. Houston, TX: Athelstan Publications.

Boud, D. ed. 1988. Developing Student Autonomy in Learning. London: Longman.

Candy, C.N. 1991. Self-direction for Lifelong Learning. California: Jossey-Bass.

Chun, D. y J. Plass. 1996. "Effects of multimedia annotations on vocabulary acquisition". The Modern Language Journal 80: 183-198.

Craik, F. I. M. y R.S. Lockhart. 1972. "Depth of processing and the retention of words in episodic memory". Journal of Experimental Psychology 104: 268-284.

Davis, N. 1980. "Facilitating effects of marginal glosses on foreign language reading". The Modern Language Journal 73 , 1: 41-48.

Egbert, J. y E. Hanson-Smith, eds. 1999. CALL Environments: Research, Practice, and Critical Issues. Bloomington, Illinois: Pantagraph Printing.

Halliday, M.A.C.K. 1979. Language as Social Semiotic. London: Edward Arnold.

Horst, M., T. Cobb. y P. Meara. 1998. "Beyond a clockwork orange: acquiring secondlanguage vocabulary through reading". Reading in a Foreign Language 11, 2: 20723.

Hubbard, P. 1992. "A methodological framework for CALL courseware development". Computers in Applied Linguistics. Eds. M. Pennington y V. Stevens. Clevedon, England: Multilingual Matters. 39-45 
Hulstijn, J.H., M. Hollander y T. Greidanius. 1996. "Incidental vocabulary learning and advanced foreign language students: the influence of marginal glosses, dictionary use, and recurrence of unknown words". Modern Language Journal 80, 3: 327-339.

Hunt, A. y D. Beglar. 1998. "Current research and practice in teaching vocabulary". The Language Teacher On-line 22: 7-11.

Hurd, S., T. Beaven y A. Ortega. 2001. "Developing autonomy in a distance language learning context: issues and dilemmas for course writers". System 29, 3: 341-355.

Knight, S. 1994. "Dictionary use while reading: the effects on comprehension and vocabulary acquisition for students of different verbal abilities". The Modern Language Journal 78, 3: 285-299.

Knowles, M.S. 1975. Self directed Learning. New York: Association Press.

Kohonen, V. 1992. "Experimental language learning: second language learning as cooperative learner education". Collaborative Learning and Teaching Ed. D. Nunnan. New York: Cambridge University Press.14-39.

Krantz, G. 1991. Learning Vocabulary in a Foreign Language. Göteborg, Sweden: Acta Universitatis Gothburgensis.

Krashen, S. 1989. "We acquire vocabulary and spelling by reading: additional evidence for the input hypothesis". Modern Language Journal 73, 4: 441-464.

Lomicka, L. 1998. "To gloss or not to gloss: an investigation of reading comprehension online". Language Learning and Technology 1, 2: 41-50.

Lyman-Hager, M., N. Davis, J. Burnett y R. Chennault. 1993. "Us Vie de Boy: interactive reading in French F.L.”. Annual Symposium on Assessment. Eds. F. Borchardt y E.M.T. Johnson. Duke University, Durham, NC: Proceedings of CALICO 1993: 9397.

Mayer, E. 1997. "Multimedia learning: are we asking the right questions?". Educational Psychologist 32, 1: 1-19.

Meskill, C. 1999. "Multimedia and language learning: assessing goals and system attributes". CALL Journal 2, 2: 11-14.

Nation, I.S.P., ed. 1994. New Ways in Teaching Vocabulary. Washington, D.C.: TESOL.

Polya, G. 1968. On Understanding Learning and Teaching Problem Solving. New York: Wiley.

Rathbone, C. H. 1971. Open Education: The Informal Classroom. New York: Citation Press.

Richards, J. y T. Rogers. 1986. Approaches and Methods in Language Teaching. Cambridge : Cambridge University Press.

Ruschoff, B. 1993 . "Language learning and information technology: state of the art". CALICO Journal 10, 3: 5-17.

Sheerin, S. 1997. "An exploration of the relationship between self-access and independent learning". Autonomy and Independence in Language Learning. Eds. P. Benson y P. Voller. London: Longman. 
Sussex, R. D. 1991. "Author, languages, authoring systems and their relation to the changing focus of computer-aides language learning”. System 19, 1: 15-27.

Wenger, E. 1987. Artificial Intelligence and Tutoring Systems: Computational and Cognitive Approaches to the Communication of Knowledge. Los Altos, CA: Morgan Kaufmann Publishers. 